\title{
Coordinated Control of Arrays of Wave Energy Devices-Benefits Over Independent Control
}

\author{
Giorgio Bacelli, Philip Balitsky, and John V. Ringwood, Senior Member, IEEE
}

\begin{abstract}
It is well known that control systems, which can modulate the power takeoff force of a wave energy device, have the capability of extending the effective device bandwidth and thereby improve energy capture. For an array of wave energy devices, there is the additional complication that each device is subject to radiated waves from other devices, which are a function of the device motion and, hence, an indirect function of each of the device controllers. This gives the possibility of enhancing the energy harvesting properties of the wave farm as a whole, by giving each controller information about the motion of other devices and employing a global performance function which allows coordinated control for the overall benefit of the farm. This paper examines the possibilities of using such coordinated control and makes conclusions on the types of devices, and types of arrays, that might benefit from such coordinated control. In addition, the overall benefit of a global array control strategy, compared to independent control of each device, is assessed against the added complexity of a coordinated control strategy.
\end{abstract}

Index Terms-Control, power optimization, power takeoff, wave energy arrays.

\section{INTRODUCTION}

$\mathbf{J}$ UST like wind farms, it is clear that commercial wave energy farms will contain multiple wave energy devices, relying on an economy of scale in reducing overall costs due to sea area leasing, moorings, electrical connections, and maintenance. However, while wind turbines can interact destructively in a collective (due to shadowing effects, etc.), wave energy devices in a farm have the possibility to interact constructively, since radiated waves from one device can reinforce the motion of another device.

However, the design of such a positively interacting collective depends on a number of factors, including the wave farm layout. In addition, the optimality of the layout (spacing, orientation, etc.), with respect to positive reinforcement, depends on sea state and incident wave direction, which are variable.

For a given wave farm layout, however, the motion of each device determines the radiated wave patterns, which are a significant function of the control strategy employed on each device. This opens the possibility that devices can, via interdevice communication, coordinate their motion to the mutual benefit of all devices in the farm, with maximum farm power production as

Manuscript received April 11, 2013; revised May 20, 2013; accepted May 29, 2013. Date of publication July 22, 2013; date of current version September 16, 2013.

The authors are with the Centre for Ocean Energy Research, National University of Ireland Maynooth, Co. Kildare, Ireland (e-mail: giorgio.bacelli@eeng. nuim.ie; philip.balitsky@eeng.nuim.ie; john.ringwood@eeng.nuim.ie)

Color versions of one or more of the figures in this paper are available online at http://ieeexplore.ieee.org.

Digital Object Identifier 10.1109/TSTE.2013.2267961

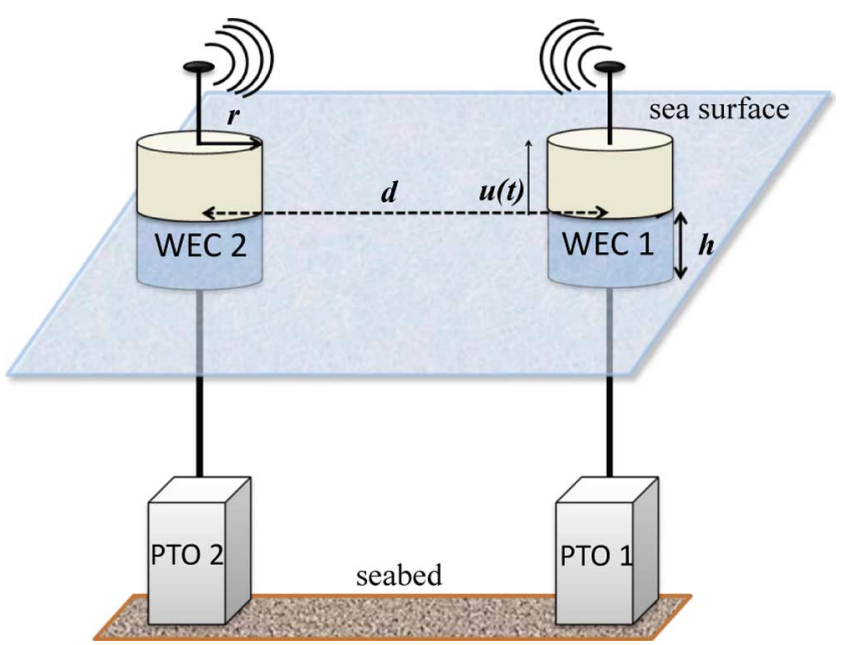

Fig. 1. Layout for a two-body array. Motion and PTO force are restricted to heave only.

an objective. Such a structure, for a simple two-device array, is shown in Fig. 1.

Wave energy arrays include both large devices (circa $1 \mathrm{MW}$ ), individually moored, and closely packed arrays of devices, which are contained within a larger superstructure. Examples of larger individual devices include Pelamis [1], Oyster [2], and Powerbuoy [3], while closely spaced arrays include those by Wavestar [4], Fred Olsen Lifesaver [5], Manchester bobber [6], and Trident Technologies [7]. In particular, compact circular arrays have been shown to be capable of extracting more energy than a single device of the same volume, and presenting a larger spectral bandwidth [8].

Original work in the area of global control of arrays was carried out by Evans [9] and Falnes [10], who developed optimal control for a number of oscillating bodies. The focus was on extending complex conjugate control to the multibody case. Since then, however, little progress has been made on optimal array control, though much research has focussed on using differential (linear) damping on individual array elements. In particular, the benefits of optimal global array control over independent control are unclear. In some cases [4], independent (optimal) control of array elements is evaluated, but there is no comparison with the global control case.

A number of studies have looked at the suboptimal case where a diagonal radiation damping matrix (considering the change in the boundary value problem, but not interdevice dynamic interactions) is employed. However, these are essentially "independent" controllers, with no interdevice communication:

1) Justino and Clement [11] show that the optimal diagonal "controller" (ignoring radiation interactions) is simply the complex conjugate of the diagonal radiation impedance 
matrix, while a similar, but simplified controller parameterization, is considered in [12]. Comparable approaches are also taken in [13] and [7].

2) A number of researchers have considered linear damping, which is optimized for each array device [14]-[17].

3) A number of studies consider linear damping where a consistent level of damping is determined for each device, but designed to maximize total array energy [13]-[15].

In this paper, two control strategies are compared for the control of WEC arrays, namely global control (GC) and independent control (IC). Both GC and IC are model-based control strategies; however, GC is based on a centralized control algorithm which uses the complete hydrodynamic model of the array whereas, with IC, each device is controlled independently using the hydrodynamic model of a single isolated device. For comparison purposes, it is assumed that the total local hydrodynamic force on each WEC can be estimated for both GC and IC, where the total hydrodynamic force is intended to be the sum of the force exerted by the incoming wave, diffraction and radiation. While we consider several layouts, separations, and body geometries, it is not the objective of this study to conclude on optimal shapes or layouts, but rather to achieve some consistency of conclusion. However, it is appreciated that WEC array performance is sensitive to both layout and device geometry [18].

In the literature, the predominant metric used to evaluate the performance of a WEC array is the $q$-factor [18], which indicated the average device performance of an array element compared to an isolated device. In this paper, however, we consider the ratio of the total array (energy) performance for the GC and IC cases. We formulate the optimal GC and IC problems in the time domain, which are then discretized and solved as nonlinear programs. While this is not necessary for the fundamental unconstrained case considered in this paper (and essentially the same results can be obtained by using the frequency domain approach originally developed by Evans [9] and Falnes $[10])$, it provides the possibility to extend the comparison to the constrained case. A preliminary analysis of the constrained GC problem was already reported in [19].

\section{Formulation OF Hydrodynamic Model}

The control systems are implemented on different array layouts and for several geometries of the WECs composing the arrays. The WECs are vertical cylinders of radius $r$, draught $h$, and the distance between their vertical axis is denoted with $d$, as depicted in Fig. 1 for the particular case of a two-body array.

\section{A. Equations of Motion}

For this work, we assume linear wave theory and noncompressible irrotational flow. Therefore, the motion of the device can be described, in the time domain, by Cummin's equation [20]

$$
M_{t} \ddot{u}(t)+B_{v} \dot{u}(t)+\int_{t_{0}}^{t} K(t-\tau) \dot{u}(\tau) d \tau+S u(t)=f(t)
$$

where $u(t) \in \mathbb{R}^{m}$ is the vector of the vertical positions of the WECs, and $m$ the number of degrees of freedom of the system, which corresponds to the number of WECs composing the array, because the motion of each device is assumed to be restricted to heave only. $M_{t}=M+m_{\infty}$, where $M \in \mathbb{R}^{m \times m}$ is the generalized mass matrix and $m_{\infty} \in \mathbb{R}^{m \times m}$ is the asymptotic values of the added mass at infinite frequency; $B_{v} \in \mathbb{R}^{m \times m}$ is the viscous damping term; $S \in \mathbb{R}^{m \times m}$ is the hydrodynamic stiffness; and $K(t) \in \mathbb{R}^{m \times m}$ is the matrix of the radiation impulse responses. The vector of external forces $f(t) \in \mathbb{R}^{m}$ is given by $f(t)=f_{e}(t)+f_{\text {pto }}(t)$, where $f_{e}(t)$ is the exciting force and $f_{\mathrm{pto}}(t)$ is the PTO force. The excitation force is calculated as $f_{e}(t)=\mathcal{F}^{-1}\{X(\omega) \eta(\omega)\}$, where $\eta(\omega)$ is the Fourier transform of the wave elevation and $X(\omega)$ is the frequency domain exciting force transfer function.

\section{B. Numerical Modeling}

The hydrodynamic coefficients $m_{\infty}$ and $K(t)$ are calculated from the frequency domain radiation impedance matrix $Z(\omega)$ by applying Ogilvie's relations [21], [22]. The matrices $X(\omega)$, $Z(\omega)$, and $S$ are then computed in the boundary element solver WAMIT [23]. This package is widely applied in studies of WEC arrays, as in [14], [16], and [17]. In WAMIT, the high order method is used and computations are performed for 160 frequencies equally spaced at intervals of $0.0151 \mathrm{rad} / \mathrm{s}$. All control simulations are implemented in MATLAB and are performed with the same frequency resolution as the WAMIT output. The software simulates the motion of the devices, the PTO forces, the instantaneous converted power, and the vertical velocities and displacements of the WECs for a representative surface elevation time-history of a given wave spectrum.

\section{Viscous Damping Approximation}

The viscous effect of a fluid on a body is usually described, under certain conditions [24], as a force proportional to the square of the relative velocity between the body and the fluid surrounding the body; that is, $f_{d}=(1 / 2) C_{d} \rho \dot{u}|\dot{u}| A$, where $\rho$ is the fluid density, $A$ is the area of the body projected onto the plane orthogonal to the velocity $\dot{u}$, and $C_{d}$ is the drag coefficient, which is obtained experimentally (see, for example, [24, Appendix 2]). Several recent studies have considered the effects of viscosity on WEC motion, such as [7], [25], and [26].

The objective of the approximation is to find a force $\hat{f}_{d}$ which is linearly proportional to the velocity and that dissipates the same amount of energy as the force $f_{d}$. This procedure is known as Lorentz linearization [27], and it has been used in the case of WECs by Folley et al. [28]. The approximation is carried out by equating the work of the nonlinear force $f_{d}$ with the work of the linear approximation $\hat{f}_{d}=B_{v} \dot{u}$ which result in $\int_{0}^{T} f_{d} \dot{u} d t=\int_{0}^{T} \hat{f}_{d} \dot{u} d t$. By means of simple manipulations, the damping coefficient $B_{v}$ can be expressed as

$$
B_{v}=\frac{C_{d} \rho A \int_{0}^{T} \dot{u}^{2}(t)|\dot{u}(t)| d t}{2 \int_{0}^{T} \dot{u}^{2}(t) d t} .
$$

For the special case of a vertical cylinder of radius $r$ oscillating sinusoidally in heave with velocity $\dot{u}(t)=U \cos (\omega t)$, the coefficient $B_{v}$ is

$$
B_{v}=\frac{4 C_{d} \rho r^{2} \omega U}{3} .
$$

Knowledge of the velocity is required to calculate the coefficient $B_{v}$, but the velocity itself depends on $B_{v}$; therefore, an 
iterative procedure has been implemented. The procedure is initiated by setting $B_{v}$ to an initial value $B_{v}^{0}$, which is not critical for the convergence because the relation between $B_{v}$ and the velocity $\dot{u}$ is monotonic ( $\dot{u}$ decreases when $B_{v}$ increases). The $i$ th iteration of the procedure is composed of two steps, which are:

1) calculate the velocity $\dot{u}^{i}$ using the value of $B_{v}^{i-1}$;

2) calculate $B_{v}^{i}$ using the velocity $\dot{u}^{i}$ and the formula (2), or (3) in the case of regular waves.

The procedure is stopped when the difference between two subsequent values of $B_{v}$ is smaller than a threshold $\delta$, that is $\mid B_{v}^{i}-$ $B_{v}^{i-1} \mid<\delta$. For any given geometry, controller type, and sea state, the coefficient $B_{v}$ is calculated by simulating an isolated device.

\section{ENERgy MAXIMIZING CONTROL}

The control problem is defined as follows: find the optimal profile for the PTO forces which maximizes the total energy absorbed by the array described by the equation of motion (1) over a time interval of length $T$. The total energy absorbed by the array is considered to be the sum of the mechanical work performed by each of the PTO forces as

$$
W=-\sum_{k=1}^{n} \int_{0}^{T} \dot{u}^{k}(t) f_{\mathrm{pto}}^{k}(t) d t
$$

where $\dot{u}^{k}(t)$ and $f_{\text {pto }}^{k}(t)$ are, respectively, the heave velocity and the PTO force of the $k$ th device.

In the ideal case, assuming the wave excitation is known completely into the future, the optimization is performed over an infinitely long time interval, that is for $T \rightarrow+\infty$; however, it has been shown [29] that a limited horizon suffices to achieve close to optimal energy absorption. As a consequence, the real-time implementation of the control algorithm can be performed in a receding horizon fashion, as described in [30], where future knowledge of either wave elevation or excitation force, up to time $T$ ahead, is obtained by prediction. Since the focus of this paper is on the control of arrays of WECS, we neglect the effects of prediction by applying the separation principle [31], which is a standard approach in control engineering, allowing the design of the optimal control to be separated from the design of the predictor; thus perfect knowledge of future wave elevation is assumed, in order to isolate the effects of control on the total energy produced.

\section{A. Discretization}

The control problem is discretized by approximating the velocity and the PTO force with a linear combination of basis functions, resulting in a finite dimension optimization problem. In this paper, trigonometric functions are chosen as basis functions, thus the PTO force and the velocity are approximated with the truncated zero-mean Fourier series

$$
\begin{aligned}
\dot{u}_{k}(t) & \approx \sum_{n=1}^{N / 2} \nu_{k}^{n, c} \cos \left(n \omega_{0} t\right)+\nu_{k}^{n, s} \sin \left(n \omega_{0} t\right) \\
f_{\mathrm{pto}_{k}}(t) & \approx \sum_{n=1}^{N / 2} \phi_{k}^{n, c} \cos \left(n \omega_{0} t\right)+\phi_{k}^{n, s} \sin \left(n \omega_{0} t\right) .
\end{aligned}
$$

The best approximation of the solution for the equation of motion (1) is sought by applying the Galerkin method, the details of which are presented in [30], and the result is the linear system

$$
G X=P+E
$$

where $X, P, E$, and $G$ are defined as

$$
X=\left[\begin{array}{l}
X_{1} \\
X_{2}
\end{array}\right] P=\left[\begin{array}{l}
P_{1} \\
P_{2}
\end{array}\right] \quad E=\left[\begin{array}{l}
E_{1} \\
E_{2}
\end{array}\right] G=\left[\begin{array}{ll}
G_{11} & G_{12} \\
G_{21} & G_{22}
\end{array}\right] .
$$

The vectors $X_{k}$ and $P_{k}$, for $k=1,2$, are the vectors of the Fourier coefficients of the velocity and PTO force of the $k$ th device, respectively, and are arranged as

$$
\begin{aligned}
X_{k} & =\left[\nu_{k}^{1, c}, \nu_{k}^{1, s}, \nu_{k}^{2, c}, \nu_{k}^{2, s}, \ldots, \nu_{k}^{N / 2, c}, \nu_{k}^{N / 2, s}\right]^{T}, \text { for } k=1,2 . \\
P_{k} & =\left[\phi_{k}^{1, c}, \phi_{k}^{1, s}, \phi_{k}^{2, c}, \phi_{k}^{2, s}, \ldots, \phi_{k}^{N / 2, c}, \phi_{k}^{N / 2, s}\right]^{T} .
\end{aligned}
$$

The elements of the vectors $E_{k}$ are the Fourier coefficients of the excitation force on the $k$ th device and are arranged in the same manner as the vectors $X_{k}$ and $P_{k}$. The matrices $G_{i j} \in \mathbb{R}^{N \times N}$ composing the matrix $G$ are block diagonal, where each of the $N / 2$ square blocks is of size two and the $l$ th block is defined as

$$
\begin{aligned}
G_{i j}^{l} & =\left[\begin{array}{cc}
D_{i j}^{l} & M_{i j}^{l} \\
-M_{i j}^{l} & D_{i j}^{l}
\end{array}\right], \quad \text { for } l=1, \ldots, \frac{N}{2} \\
D_{i j}^{l} & =R_{i j}\left(l \omega_{0}\right)+B_{v_{i j}} \\
M_{i j}^{l} & =l \omega_{0}\left(M_{i j}+m_{i j}\left(l \omega_{0}\right)\right)-\frac{S_{i j}}{\left(l \omega_{0}\right)} .
\end{aligned}
$$

$B_{i j}, M_{i j}$, and $S_{i j}$ are, respectively, the elements of the matrices $B, M$, and $S$, while $R_{i j}(\omega)$ and $m_{i j}(\omega)$ are the elements of the radiation impedance matrix $Z(\omega)$, which is computed by WAMIT and is defined as $Z(\omega)=R(\omega)+i \omega m(\omega)$.

\section{B. Global Control}

The control system of the GC strategy is aware of the whole configuration of the array; the resulting optimization problem is defined by the cost function $W=-(T / 2) P^{T} X$, which is obtained by substituting (5) and (6) into the definition of the total absorbed energy in (4). If $G$ is nonsingular, the cost function can be expressed as a function of $P$ by solving (7) w.r.t. $X$, and the coefficients $P^{\star}$ of the optimal PTO forces that maximize the absorbed energy for the array are obtained by solving the optimization problem ${ }^{1}$

$$
P^{\star}=\arg \max _{P}-P^{T} G^{-1} P-P^{T} G^{-1} E .
$$

\section{Independent Control}

For the IC case, it is assumed that each device is equipped with its own controller and an excitation force estimator. It is also assumed that no communication occurs between the devices, and each controller uses the model of a single isolated device. That is, the control system of each device uses the model

\footnotetext{
${ }^{1}$ The term $T / 2$ is a positive scaling factor that can be neglected for the calculation of the optimal PTO force because the vector $P^{\star}$ which maximizes $W=-(T / 2) P^{T} X$ also maximizes the cost function $\bar{W}=-P^{T} X$.
} 
(1), where the variables $u(t)$ and $f(t)$ are scalars and where $M_{t}, B, K(t), S$ are also scalars and they are the parameters of a single isolated device with the same shape and dimensions of the devices composing the corresponding array. For the example case of an array composed of two WECs (as shown in Fig. 1), the controllers of devices 1 and 2 use, respectively, the discretized models

$$
\begin{aligned}
G_{s} X_{1} & =P_{1}+\bar{E}_{1} \\
G_{s} X_{2} & =P_{2}+\bar{E}_{2}
\end{aligned}
$$

where $G_{s} \in \mathbb{R}^{N \times N}$ is analogous to the matrix $G$ in the approximated equation of motion of the array in (7). However, in this case, $G_{s}$ is calculated using the hydrodynamic coefficients of a single isolated device. In particular, $G_{s}$ is a block diagonal matrix with square blocks of size two, and is constructed in the same manner as the diagonal blocks $G_{i i}$ of the matrix $G$, as described in (9) for $i=j$, with the difference being that the elements $D_{i j}^{l}$ and $M_{i j}^{l}$ of $G_{s}$ are calculated using the radiation impedance matrix of a single isolated device.

$\bar{E}_{k}$ is the excitation force measured by the estimator on WEC $k$; more specifically, $\bar{E}_{1}=E_{1}-G_{12} X_{2}$ and $\bar{E}_{2}=E_{2}-G_{21} X_{1}$. In fact, it is assumed that the excitation force estimator on each device is not capable of discerning the excitation force due to incoming waves from the radiation generated by other bodies; therefore, the estimator provides a signal which is the sum of the radiation force caused by other bodies $\left(G_{12} X_{2}\right.$ and $\left.G_{21} X_{1}\right)$ and excitation from incoming waves $\left(E_{1}\right.$ and $\left.E_{2}\right)$.

Each of the independent controllers calculates the optimal PTO force that maximizes the energy absorbed by the corresponding WEC using the models in (11) and (12). The optimal PTO forces $\left(P_{1}^{*}\right.$ and $\left.P_{2}^{*}\right)$ are the solutions of the optimization problems

$$
\begin{aligned}
P_{1}^{*} & =\arg \max _{P_{1}} W_{1} \\
& =\arg \max _{P_{1}}-P_{1}^{T} G_{s}^{-1} P_{1}-P_{1}^{T} G_{s}^{-1} \bar{E}_{1} \\
P_{2}^{*} & =\arg \max _{P_{2}} W_{2} \\
& =\arg \max _{P_{2}}-P_{2}^{T} G_{s}^{-1} P_{2}-P_{2}^{T} G_{s}^{-1} \bar{E}_{2}
\end{aligned}
$$

the cost functions of which are the energy absorbed by each device, that is $W_{1}=-P_{1}^{T} X_{1}$ and $W_{2}=-P_{2}^{T} X_{2}$. However, the solutions of the optimization problems in (13) and (14) are coupled because $P_{1}^{*}$ depends on $\bar{E}_{1}$, which is function of the velocity of body $2\left(X_{2}\right)$, and vice versa; the problem is then effectively solved iteratively. The initial condition is considered to be with the PTOs switched OFF $\left(P_{1}=P_{2}=0\right)$. The velocities $X_{1}$ and $X_{2}$ are then calculated by means of the equation of motion (7) and the controller calculates the PTO forces by solving the optimization problems (13) and (14). When the PTO forces are applied to the WECs, the new velocities are calculated again, using (7), and the process is repeated. The computations performed by the controller at the $n$th step of the iteration are

$$
\begin{aligned}
X^{n} & =G^{-1}\left(\hat{P}^{n-1}+E\right) \\
\hat{P}_{1}^{n} & =\arg \max _{P_{1}}-P_{1}^{T} G_{s}^{-1} P_{1}-P_{1}^{T} G_{s}^{-1}\left(E_{1}-G_{12} X_{2}^{n}\right) \\
\hat{P}_{2}^{n} & =\arg \max _{P_{2}}-P_{2}^{T} G_{s}^{-1} P_{2}-P_{2}^{T} G_{s}^{-1}\left(E_{2}-G_{21} X_{1}^{n}\right)
\end{aligned}
$$
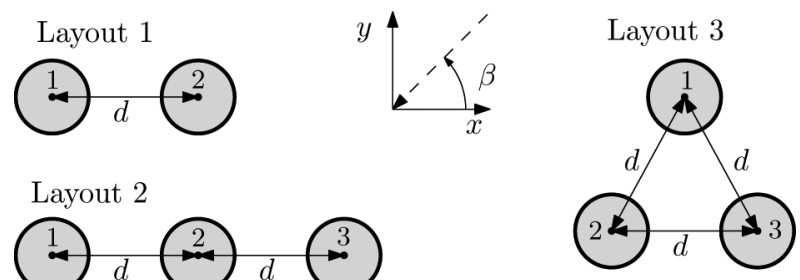

Layout 2

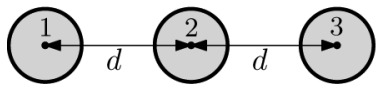

Fig. 2. Top view of array layouts and of incident waves angle $\beta$.

TABLE I

SET OF GEOMETRIES CONSIDERED

\begin{tabular}{c||ccc}
\hline WEC Geometry & 1 & 2 & 3 \\
\hline \hline Radius $r(m)$ & 4 & 5 & 6.25 \\
Draught $h(m)$ & 10 & 6 & 4 \\
Resonance $T_{r}(s)$ & 7.1 & 5.9 & 5.4 \\
\hline
\end{tabular}

where $n>1, \hat{P}^{0}=\mathbf{0}$ and $\hat{P}^{n}, \hat{P}_{1}^{n}, \hat{P}_{2}^{n}, X^{n}, X_{1}^{n}, X_{2}^{n}$ are defined according to (8). The iteration stops when the PTO forces approach their asymptotic values, which is implemented by the condition $\left\|P_{k}^{n}-P_{k}^{n-1}\right\|<\varepsilon \forall k=1,2$.

\section{RESULTS}

We consider three possible array layouts (Fig. 2): layout 1 is composed of two heaving cylinders, layout 2 is a linear array of three WECs, while layout 3 is composed of three WECs placed at the vertices of an equilateral triangle. For each layout, we have chosen 24 values of the interbody spacing $d$, logarithmically spaced between $2.2 r$ and $10 r$. Three device geometries for the WECs composing each array have been simulated, each of which has approximately the same volume $\left(\approx 160 \pi \mathrm{m}^{3}\right)$ but different resonant periods; Table I lists the radii $r$, draughts $h$, and resonant period $T_{r}$ for each device.

The optimal control laws for each WEC in the case of IC are obtained by iteration, and it is assumed that, between consecutive iterations, there is enough time for the waves radiated from a device to reach all the other devices, and enough time for the estimator and predictor on each WEC to build a reliable forecast of the incoming waves (reach quasi-steady-state). While this is not feasible in practice, it provides a best-case scenario for the IC case, providing an upper performance bound for the IC case. However, the comparison between the GC and the ICs is also carried out by considering only the first iteration of the ICs, which start from an initial condition where each PTO is switched OFF. The comparison of the GC with first iteration of the ICs is interesting from a practical point of view, since it highlights the effect of the PTO (and, therefore, the control system) on the interaction between devices.

Each of the three array layouts depicted in Fig. 2 has been simulated for all three WEC geometries in Table I. Both GC and ICs have been computed for each of the resulting nine possible arrays with interbody spacing ranging between $2.2 r$ and $10 r$, and considering four Bretschneider spectra with $H_{s}=1 \mathrm{~m}$ and $T_{p}=\{6,8,10,12\} \mathrm{s}$. The parameters $\omega_{0}$ and $N$ for the discretization of the control problem are $N=160$ and $\omega_{0}=$ $2 \pi / 200 \mathrm{rad} / \mathrm{s}$, respectively, while the thresholds $\delta$ and $\beta$ for the adaptive approximation of the viscous damping (Section II-C) and the ICs (Section III-C), respectively, are $\delta=1$ and $\varepsilon=10$. 

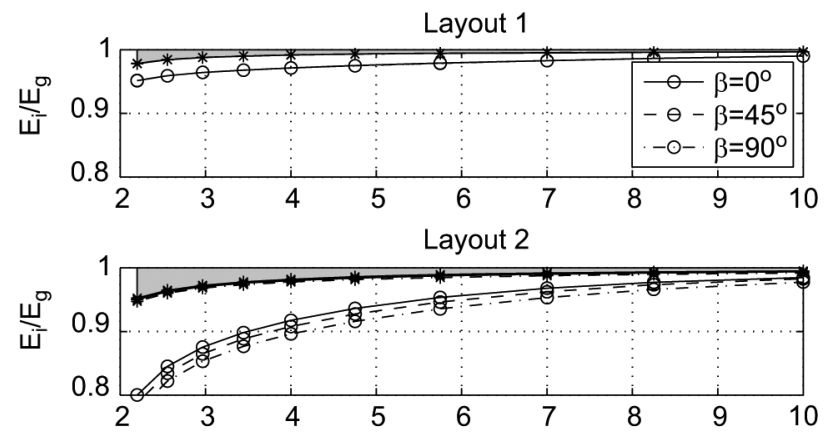

Layout 3

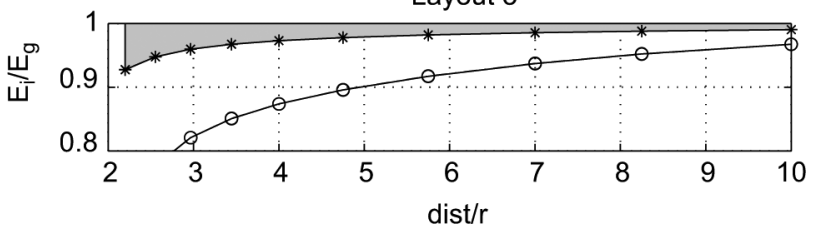

Fig. 3. Relative performance of IC and GC for geometry $1, T_{p}=12 \mathrm{~s}(\lambda=$ $225 \mathrm{~m})$. The mark $*$ identifies the asymptotic values of $E_{i} / E_{g}$; the mark o identifies the first iteration $E_{i}^{1} / E_{g}$.

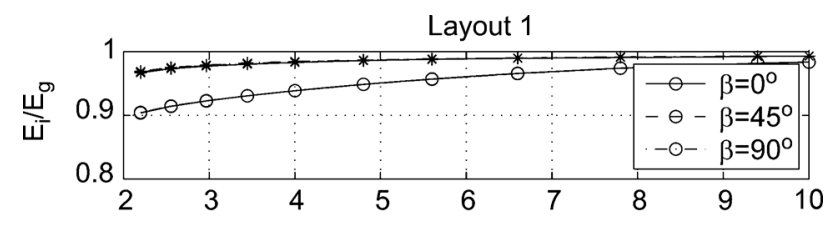

Layout 2

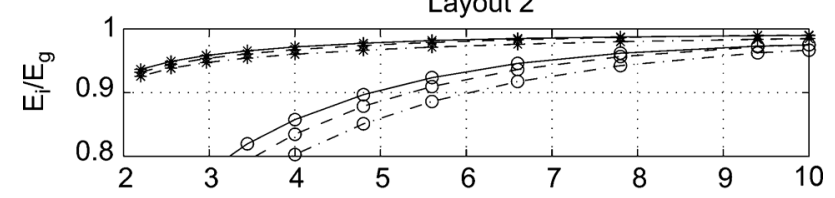

Layout 3

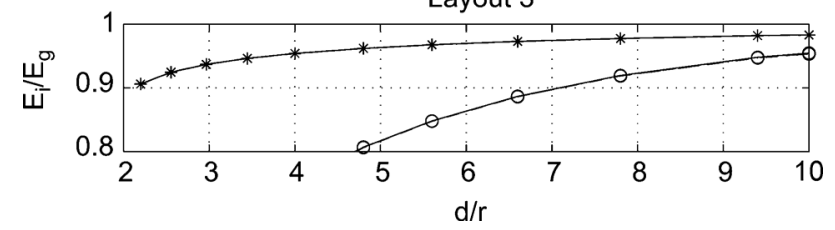

Fig. 4. Relative performance of IC and GC for geometry $2, T_{p}=12 \mathrm{~s}(\lambda=$ $225 \mathrm{~m})$.

The patterns exhibited by the results are illustrated by the representative cases depicted in Figs. 3-5, which show the ratio between the energy absorbed using IC and GC $\left(E_{i} / E_{g}\right)$. In addition, Tables II-IV summarize the totality of the results for the different geometries, with the table entries representing the integral of the difference between the (ideal) IC and GC cases $\left(\int_{2.2}^{10}\left(1-E_{i} / E_{g}\right) d(d / r)\right)$, as highlighted by the shaded area in Fig. 3. In Figs. 3-5, the asterisk marks denote curves corresponding to the asymptotic value of the IC, that is when the control algorithm reaches (the ideal situation of) convergence in the calculation of the control law, while the circle marks denote the curves corresponding to the first iteration.

As expected, the GC always performs better than the ICs in terms of absorbed energy, with the difference decreasing monotonically with an increase in the separation distance. Although the difference between GC and ICs, for the ideal IC case, is generally smaller than $5 \%-10 \%$, the relative performance is considerably larger when a comparison is made with the first iteration
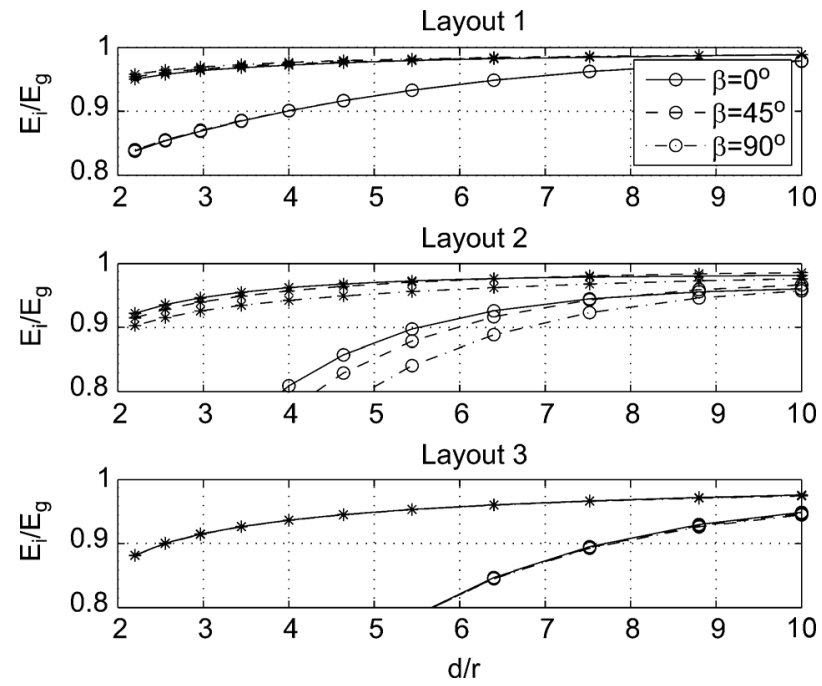

Fig. 5. Relative performance of IC and GC for geometry $3, T_{p}=12 \mathrm{~s}(\lambda=$ $225 \mathrm{~m})$.

TABLE II

VALUES OF $\int_{2.2}^{10}\left(1-E_{i} / E_{g}\right) d(d / r)$ FOR GEOMETRY 1

\begin{tabular}{l|cccc}
\hline & $T_{p}=6 s$ & $T_{p}=8 s$ & $T_{p}=10 s$ & $T_{p}=12 s$ \\
\hline Layout 1 & 0.072 & 0.068 & 0.064 & 0.054 \\
Layout 2 & 0.113 & 0.122 & 0.130 & 0.121 \\
Layout 3 & 0.200 & 0.188 & 0.188 & 0.170 \\
\hline
\end{tabular}

TABLE III

VALUES OF $\int_{2.2}^{10}\left(1-E_{i} / E_{g}\right) d(d / r)$ FOR GEOMETRY 2

\begin{tabular}{l|cccc}
\hline & $T_{p}=6 s$ & $T_{p}=8 s$ & $T_{p}=10 s$ & $T_{p}=12 s$ \\
\hline Layout 1 & 0.132 & 0.135 & 0.123 & 0.102 \\
Layout 2 & 0.217 & 0.225 & 0.219 & 0.200 \\
Layout 3 & 0.310 & 0.313 & 0.307 & 0.279 \\
\hline
\end{tabular}

TABLE IV

VALUES OF $\int_{2.2}^{10}\left(1-E_{i} / E_{g}\right) d(d / r)$ FOR GEOMETRY 3

\begin{tabular}{l|cccc}
\hline & $T_{p}=6 s$ & $T_{p}=8 s$ & $T_{p}=10 s$ & $T_{p}=12 s$ \\
\hline Layout 1 & 0.190 & 0.207 & 0.191 & 0.160 \\
Layout 2 & 0.324 & 0.335 & 0.312 & 0.281 \\
Layout 3 & 0.429 & 0.433 & 0.420 & 0.387 \\
\hline
\end{tabular}

of the IC. In addition, the performance of the ICs degrades when the number of bodies in the array increases.

Comparing Figs. 3-5, it is evident that the ICs suffer a degradation in performance for devices with stronger radiative properties. In fact, at any distance $d$, the ratio $E_{i} / E_{g}$ for geometry 3 (Fig. 5) is smaller than $E_{i} / E_{g}$ for geometry 2 (Fig. 4), which is a flatter device compared with geometry 3 ; in turn, the $E_{i} / E_{g}$ for geometry 2 is smaller than $E_{i} / E_{g}$ for geometry 1 (Fig. 3), which is flatter than geometry 2. By flatter, we mean that $r / h$ is larger, with resulting stronger radiative properties. Tables II-IV show how the relative behaviors of the GC and ICs are affected by the sea state, for different geometries. For layout 1, composed of two WECs, the relative performance of the asymptotic value of the IC case with respect to the GC case is not significantly 


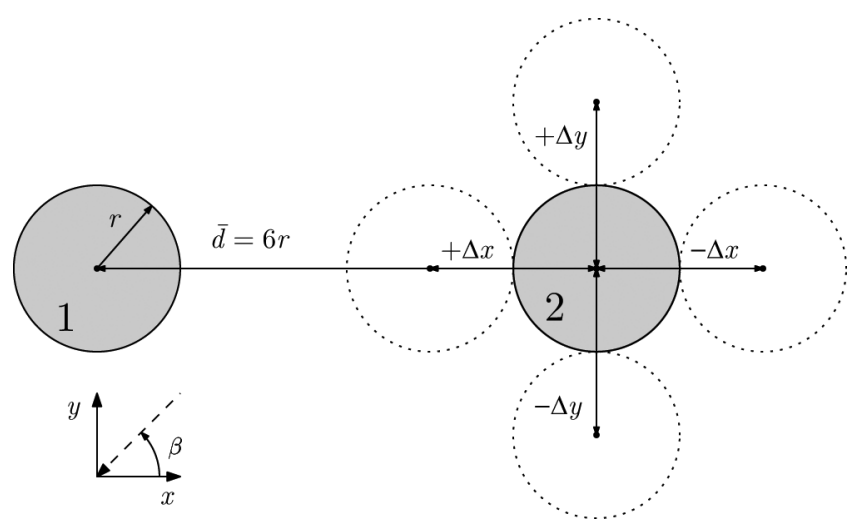

Fig. 6. Top view of the array layout for the sensitivity analysis. The nominal distance $\bar{d}$ between the devices is $\bar{d}=6 r$. The devices in the nominal position are colored in grey, while the dotted white circles denote the positions of the devices corresponding to the maximum offsets $( \pm \Delta x, \pm \Delta y=2 r)$.

affected by sea state, while more significant variations exist for layouts 2 and 3 .

For layouts 2 and 3 , the patterns in the $E_{i} / E_{g}$ curves are still similar to the case of the layout 1 ; however, the dependency on the heading angle emerges for layout 2, especially when considering the first iteration of the IC. This phenomenon shows that the performance of the ICs is affected by the wave heading angle only in the case of the extended linear array (layout 2).

\section{SENSITIVITY ANALYSIS}

One potential drawback of the GC is the reliance on a more complex model, with particular emphasis on interdevice interactions. We could, therefore, expect that variations in interdevice separation (for slack-moored devices) might introduce a modeling error which may impact the performance of the GC.

While some sensitivity analysis has been previously performed for WEC arrays, these studies mainly concerned sensitivity of suboptimal control to mis-tuning of the damping [12], [16], or the sensitivity of the suboptimal damping to sea state. In [32], the effect of the uncertainty in the position of WECs on the interaction factor for an array has been considered from a probabilistic point of view, using individual linear damping. However, these studies do not employ a "fully aware" hydrodynamic model, where off-diagonal damping terms form an integral part of the control model.

Here, we examine the sensitivity of the relative IC/GC performance with respect to position variation of an array element; in particular, we consider layout 1 , which is composed of two WECs, and we study the relative performance of the GC compared to the IC in terms of absorbed energy, that is $E_{i} / E_{g}$. Fig. 6 depicts the configuration that has been used for the sensitivity analysis; body 1 is held fixed while body 2 is displaced around its nominal position, for which $\bar{d}=6 r$. The offsets $\Delta x$ and $\Delta y$ have been chosen as uniformly distributed between $-2 r$ and $2 r$, for a total of 21 values including zero, with an increment of $0.2 r$.

The ICs, by design, are not concerned with the position of the WECs, thus the calculation of the optimal profile of the PTO forces and energy absorbed is performed as described in Section III-C.

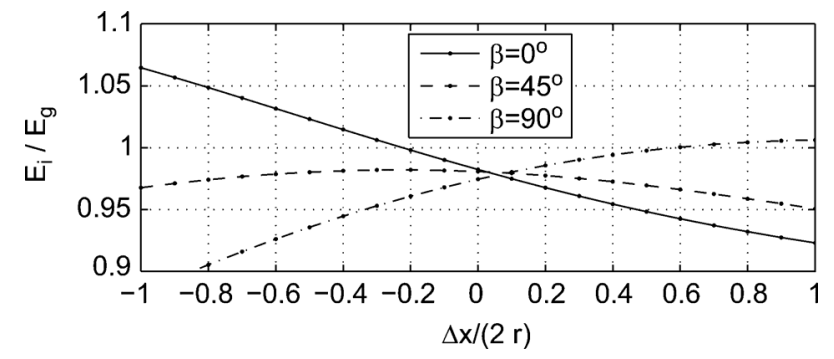

Fig. 7. Sensitivity to offset along $x$ for geometry 3 , with $T_{p}=6 \mathrm{~s}, \lambda=56 \mathrm{~m}$. Correct excitation force estimation.
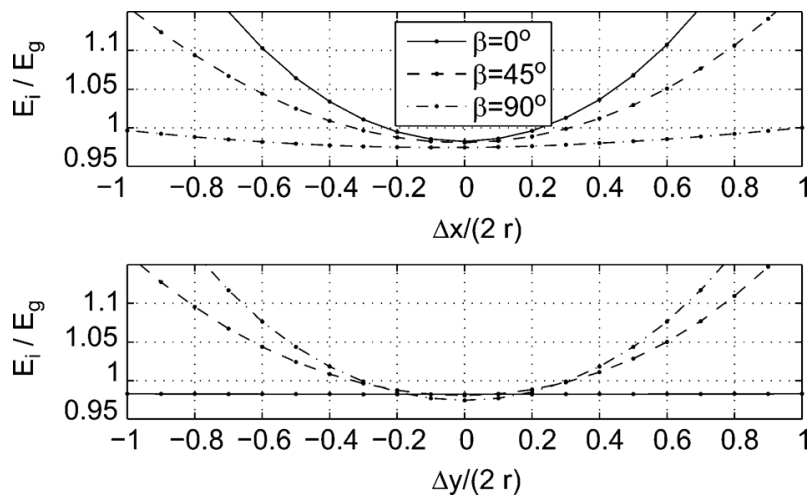

Fig. 8. Sensitivity to offset along $x$ and $y$ for geometry 3 for $T_{p}=6 \mathrm{~s}, \lambda=$ $56 \mathrm{~m}$. Incorrect excitation force estimation.

Two cases have been considered for the GC, depending on the availability of correct estimation of the excitation force. In the first case, the optimal profile of the PTO force is calculated using correct values of the excitation force; this can be the situation, for example, where the estimation of the excitation forces are performed using sensors on board the WEC. Although the real position of the device is unknown, the excitation forces are correctly estimated (neglecting other phenomena) because the measurements used for the estimation are taken at the correct location. The second case introduces an error in the estimation of the excitation force which can be due to the fact, for example, that the wave field is measured using wave data buoys located at a certain known distance from the nominal position of the WECs. In this situation, the algorithm for the estimation of the excitation forces can only provide values related to the nominal positions of the WECs.

The sensitivity results are displayed using some indicative detailed plots (Fig. 7 for the case of correct excitation force estimation and Fig. 8 for the case of incorrect excitation force estimation), while summary results are presented in Tables V-VII for the case of incorrect estimation of the excitation force. The table entries show the maximum deviation of $E_{i} / E_{g}$ due to the perturbation (normally achieved at $\Delta x / 2 r=1$ ) and only variations in $\Delta x$ are shown, due to the virtual symmetry between the cases of $[\beta=0, \Delta x \neq 0]$ and $\left[\beta=90^{\circ}, \Delta y \neq 0\right]$. Significant symmetry is also shown for $\beta=45^{\circ}$.

\section{A. Correct Estimation of the Excitation Force}

The optimal PTO force profile calculated by the GC when the WEC 2 is in a displaced position is obtained by using (7) as the controller model. However, the excitation force vector $E$ is replaced by $E_{o}$, which is the correct value of the excitation force at the exact location of the device. Thus, in this case, the 
TABLE V

GEOMETRY $1 ; \max _{\Delta x}\left(E_{i} / E_{g}\right)-E_{i} /\left.E_{g}\right|_{\Delta x=0}$

\begin{tabular}{l|cccc}
\hline & $T_{p}=6 s$ & $T_{p}=8 s$ & $T_{p}=10 s$ & $T_{p}=12 s$ \\
\hline$\beta=0^{\circ}$ & 0.096 & 0.035 & 0.018 & 0.011 \\
$\beta=45^{\circ}$ & 0.039 & 0.016 & 0.009 & 0.006 \\
$\beta=90^{\circ}$ & 0.003 & 0.002 & 0.002 & 0.002 \\
\hline
\end{tabular}

TABLE VI

GEOMETRY $2 ; \max _{\Delta x}\left(E_{i} / E_{g}\right)-E_{i} /\left.E_{g}\right|_{\Delta x=0}$

\begin{tabular}{l|cccc}
\hline & $T_{p}=6 s$ & $T_{p}=8 s$ & $T_{p}=10 s$ & $T_{p}=12 s$ \\
\hline$\beta=0^{\circ}$ & 0.211 & 0.067 & 0.031 & 0.018 \\
$\beta=45^{\circ}$ & 0.092 & 0.030 & 0.015 & 0.009 \\
$\beta=90^{\circ}$ & 0.012 & 0.007 & 0.005 & 0.003 \\
\hline
\end{tabular}

TABLE VII

GEOMETRY $3 ; \max _{\Delta x}\left(E_{i} / E_{g}\right)-E_{i} /\left.E_{g}\right|_{\Delta x=0}$

\begin{tabular}{l|cccc}
\hline & $T_{p}=6 s$ & $T_{p}=8 s$ & $T_{p}=10 s$ & $T_{p}=12 s$ \\
\hline$\beta=0^{\circ}$ & 0.390 & 0.119 & 0.049 & 0.027 \\
$\beta=45^{\circ}$ & 0.200 & 0.057 & 0.024 & 0.014 \\
$\beta=90^{\circ}$ & 0.026 & 0.013 & 0.008 & 0.005 \\
\hline
\end{tabular}

error is only in the matrix $G$, which is built using hydrodynamic coefficients of the devices in their nominal position, instead of using the exact position. The optimal profile for the PTO force $P_{o}^{\star}$ is then the solution of the quadratic optimization problem defined by (10), where $E$ is replaced by $E_{o}$, that is

$$
P_{o}^{\star}=\arg \max _{P}-P^{T} G^{-1} P-P^{T} G^{-1} E_{o}
$$

and the energy absorbed by the array is calculated as $W_{o}=$ $P^{* T} X_{o}$, where the vector of the exact velocities of the devices $X_{o}$ is calculated using (7) as $X_{o}=G_{o}^{-1}\left(P_{o}^{*}+E_{o}\right)$, where $G_{o}$ is built using the hydrodynamic coefficients of the WECs in their exact locations.

The relative performance of the GC with respect to ICs, measured with the ratio $E_{i} / E_{g}$, is not sensitive to variations in the position along the $y$ axis, i.e., to position variations when the WEC moves orthogonally to the plane passing through the axes of the cylinders (the $x z$ plane). In general, it has been found that the relative performance of the GC with respect to the ICs is less sensitive to position offsets when the sea state has a larger $T_{p}$.

It has also been observed that for all the sea states and geometries, the GC and ICs perform similarly when the incident wave angle is $45^{\circ}$; in fact, for any value of $\Delta x$, the line corresponding to $\beta=45^{\circ}$ is always close to the value that $E_{i} / E_{g}$ takes for $\Delta x=0$.

\section{B. Incorrect Estimation of the Excitation Force}

In this case, the GC optimal PTO profile $P_{o e}^{\star}$ is calculated by solving the quadratic optimization problem (10), with both $E$ and $G$ referring to the nominal position of the devices. The energy absorbed by the array is then calculated as $W_{o e}=P_{o e}^{* T} X_{o e}$, where $X_{o e}=G_{o}^{-1}\left(P_{o e}^{*}+E_{o}\right)$, with $G_{o}$ and $E_{o}$ defined as in Section V-A.

Fig. 8 shows the comparison of performance between GC and IC, for Geometry 3, with $T_{p}=6 \mathrm{~s}$. The GC performs progressively worse than the IC with increasing device displacement, as the curves $E_{i} / E_{g}$, for any heading angle $\beta$, always reach their minimum for $\Delta x=0$ and $\Delta y=0$.

Similar to the case where the estimation of the excitation force is correct, the degradation in performance of GC with respect to IC is smaller for longer wavelengths (see Tables V-VII) and for devices with smaller radiation characteristics (compare Table V to Table VII).

When the estimation of the excitation force is affected by the error due to the position offset of one device, the relative performance of the GC with respect to the ICs degrades significantly when the offset takes place in the direction of the incoming waves. Fig. 8 , for example, shows that when $\beta=0^{\circ}, E_{i} / E_{g}$ is not affected by offsets along $y$, but is notably sensitive to displacements along $x$. Conversely, when $\beta=90^{\circ}, E_{i} / E_{g}$ is strongly affected by variation of position along $y$ and it is not sensitive along the $x$ direction. The explanation of this effect can be related to the fact that a displacement of the body in the direction of the incoming waves corresponds to a phase shift in the excitation force, while a displacement in the direction orthogonal to the incoming waves only affect the diffraction component. In particular, the Froude-Krylov force is constant on a direction orthogonal to the wave direction (plane wave case), while the diffraction force depends on the layout of the array and on the geometry of the devices. The effect of a small displacement along $y$ on the diffraction component is negligible because the distance $d$ between the bodies remains unaltered, that is $d \approx \bar{d}$. However, a displacement along $x$ might affect the diffraction component because the variation in the distance between the devices is equal to the magnitude of the offset $\Delta x$, that is $|d-\bar{d}|=|\Delta x|$.

Fig. 8 allows us to discern between the effects of the diffraction and the Froude-Krylov forces on the relative performance between GC and ICs. In particular, the curve corresponding to $\beta=0^{\circ}$ is perfectly horizontal when the displacement occurs along $y$, where variations of both diffraction and Froude-Krylov forces are negligible. For $\beta=90^{\circ}$, the curve corresponding to displacements along $y$ is not constant, and its variation is due to diffraction only because, in this case, the Froude-Krylov component is constant along $y$.

\section{DISCUSSION}

One striking feature of the results is the characteristic "asymptotic" convergence of $E_{i} / E_{g}$ to 1 , as $d / r$ becomes large. This can be explained by the reduction in interaction effects as the separation distance between array elements increases.

In [33], Babarit showed that, for a two-body array of heaving or surging WECs aligned with the direction of the incoming waves and large $d$, when radiation is negligible, the alteration of the energy absorption due to wave interaction effects decreases asymptotically with the square root of the separating distance. A similar discussion is carried out in [32], where further development accounts for the effects of radiation at closer distances; although the authors provide a model for the approximation of the interaction factor as function of the separating distance, the effect of radiation on the interaction between WECs was not isolated from the effect of diffraction. Separation between the effects of diffraction and radiation on the energy absorption have 


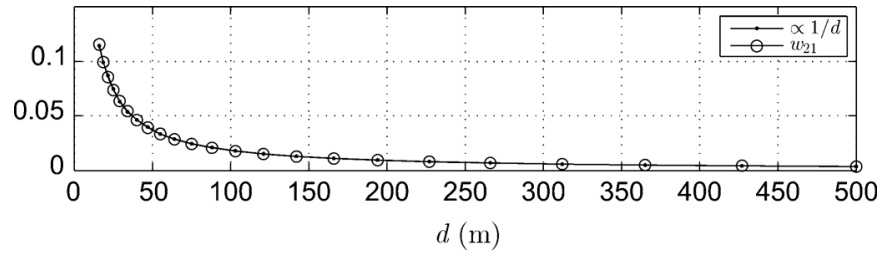

Fig. 9. Interaction factor $w_{21}$ for geometry 3 as a function of $d$.

been considered in Babarit et al. [34], where the problem has been studied by considering a two-body array and keeping one device fixed, inhibiting radiation.

Consider a two-body array with no incoming waves, with the first device (WEC 1) induced to oscillate by means of a prespecified PTO force $\left(P_{1}\right)$, and the second device (WEC 2) to be optimally controlled so as to absorb the maximum amount of energy radiated by the first device. As expected, from analytical considerations, the maximum amount of energy transferred from the first device to the second is inversely proportional to the distance $d$. The first step to show this effect is to define $H$, if $G$ is nonsingular, as

$$
H=G^{-1}=\left[\begin{array}{ll}
H_{11} & H_{12} \\
H_{21} & H_{22}
\end{array}\right] .
$$

From the discretized equation of motion (7), the energy absorbed by WEC 2 , denoted as $J_{2}$, is

$$
J_{2}=P_{2}^{T} X_{2}=P_{2}^{T} H_{21} P_{1}+P_{2}^{T} H_{22} P_{2} .
$$

For this two-body case, it can be seen that the symmetric part of $G$ (the symmetric part of $G$ is $\left(G+G^{T}\right) / 2$ ) is positive definite, thus also the symmetric parts of $H$ and, given the structure of $G, H_{12}$ is positive definite, and (16) is a convex quadratic function of $P_{2}$. Therefore, for any given value of the PTO force $P_{1}$, the optimal value of the PTO force $P_{2}$ that maximizes $J_{2}$ is $P_{2}^{*}=\left(G+G^{T}\right)^{-1} H_{21} P_{1}$.

The "radiation interaction factor" $w_{21}$, defined as the ratio of the energy delivered to the system by means of the mechanical work of the PTO on WEC 1 and the energy recovered by means of the optimally controlled WEC 2 , is $w_{21}=J_{2} / J_{1}=$ $P_{2}^{T} X_{2} / P_{1}^{T} X_{1}$. Using (7) and (8), and some further manipulation, $w_{21}$ can be evaluated as

$$
w_{21}=\frac{P_{1}^{T} H_{21}^{T}\left(\bar{H}_{2}^{T} H_{22} \bar{H}_{2}+\bar{H}_{2}^{T}\right) H_{21} P_{1}}{P_{1}^{T}\left(H_{11}+H_{12} \bar{H}_{2} H_{21}\right) P_{1}}
$$

where $\bar{H}_{2}=\left(H_{22}+H_{22}^{T}\right)^{-1}$.

Fig. 9 shows that $w_{21}$ is inversely proportional to the interbody distance $d$. In particular, the curve marked with circles (o) depicts the values of $w_{21}$ for an array composed of two devices of geometry 3 as a function of the interbody distance $d$, where $P_{1}$ has been chosen as a random vector. The curve marked with dots $(\cdot)$ is obtained by fitting the values of $w_{21}$ with a function $f(d)=\alpha / d$, where the value of $\alpha$ is obtained using least squares.

\section{CONCLUSION}

The results in Section IV clearly indicate that a significant performance improvement (up to $10 \%$, or more) can be ob- tained using global control of arrays, compared with independent control. Considering the more realistic case where the ICs will not have time to fully converge on a quasi-steady-state (which assumes that the sea state is strictly stationary), significantly greater benefits of GC are indicated, with improvements of greater than $20 \%$ possible for small $d$. The benefits of $\mathrm{GC}$ are greatest for layouts which have maximum device interaction (e.g., layout 3 in our analysis) and devices which have strong radiation properties (e.g., geometry 3 , which has a large radius/draught ratio).

The degree of benefit of the GC over the ICs, as expected, diminishes as the interaction between devices decreases. Therefore, the greatest benefit of GC is likely to be manifest for closely packed arrays of WECs, such as Wavestar [4], Fred Olsen Lifesaver [5] , Manchester bobber [6], and Trident Technologies [7] systems. In addition, since the individual devices within these systems are held within a relatively rigid framework, a GC is not likely to suffer from performance degradation due to incorrect specification of the position of the individual devices, following the analysis in Section V.

For GC, some extra complexity and computation is required. However, the addition of hardware components is minimal, though some interdevice communication is required (which could be achieved wirelessly, or performed in tandem with the electrical connections). The main additional complexity is in the form of the hydrodynamic model and, consequently, the control equations. Given a potential $10 \%$ improvement in energy converted, this would seem to be a small price to pay, considering the considerable capital cost of a wave energy array system and must be seriously considered as a solution component in the challenge to make wave energy economic.

A future study needs to be conducted to determine the effect of force and amplitude constraints on the efficacy of global array control. A preliminary analysis for the amplitude constrained case is given in [19]. In addition (or introducing it as an extra constraint), power smoothing objectives may be also be possible to achieve using global control.

\section{REFERENCES}

[1] M. Carcas, The Pelamis wave energy converter, Ocean Power Delivery Ltd., 2010, vol. 10.

[2] T. Whittaker and M. Folley, "Nearshore oscillating wave surge converters and the development of Oyster," Philosophical Trans. Royal Soc. A. Math., Phys., Eng. Sci., vol. 370, no. 1959, pp. 345-364, 2012.

[3] M. Draper, "More than just a ripple: Ocean power technologies sets its sights high," Refocus, vol. 7, no. 1, pp. 54-56, 2006.

[4] R. H. Hansen and M. M. Kramer, "Modelling and control of the Wavestar prototype," in Proc. 9th Eur. Wave and Tidal Energy Conf. (EWTEC), Southampton, U.K., 2011.

[5] C. M. Sandvik, "Wave-to-Wire Model of the Wave Energy Converter Bolt2: Control and Power Extraction with an All-Electric Power Take-Off System," Ph.D. dissertation, Norwegian Univ. of Science and Technology, Trondheim, Norway, 2012.

[6] X. Garnaud and C. C. Mei, "Wave-power extraction by a compact array of buoys," J. Fluid Mechanics, vol. 635, pp. 389-413, 2009.

[7] R. Antonutti and G. E. Hearn, "Optimisation of point-absorber arrays," in Proc. 9th Eur. Wave and Tidal Energy Conf. (EWTEC), Southampton, U.K., 2011.

[8] X. Garnaud and C. Mei, "Comparison of wave power extraction by a compact array of small buoys and by a large buoy," IET Renew. Power Generat., vol. 4, pp. 519-530, Nov. 2010.

[9] D. Evans, "Some theoretical aspects of three-dimensional wave-energy absorbers," in Proc. 1st Symp. Wave Energy Utilization, Gothenburg, Sweden, 1979, Chalmers University of Technology. 
[10] J. Falnes, "Radiation impedance matrix and optimum power absorption for interacting oscillators in surface waves," Appl. Ocean Res., vol. 2, pp. 75-80, Apr. 1980.

[11] P. A. Justino and A. Clément, "Hydrodynamic performance for small arrays of submerged spheres," in Proc. 5th Eur. Wave and Tidal Energy Conf. (EWTEC), Cork, Ireland, 2003, pp. 266-273.

[12] B. Child and V. Venugopal, "Non-optimal tuning of wave energy device arrays," in Proc. 2nd Int. Conf. Ocean Energy (ICOE), Brest, France, 2008.

[13] S. Bellew, T. Stallard, and P. Stansby, "Optimisation of a heterogeneous array of heaving bodies," in Proc. 8th Eur. Wave and Tidal Energy Conf. (EWTEC), Uppsala, Sweden, 2009, pp. 519-527.

[14] G. De Backer, M. Vantorre, C. Beels, J. De Rouck, and P. Frigaard, "Power absorption by closely spaced point absorbers in constrained conditions," IET Renew. Power Generat., vol. 4, pp. 579-591, Nov. 2010.

[15] P. Ricci, J.-B. Saulnier, and A. F. de O. Falcão, "Point-absorber arrays: A configuration study off the Portuguese west-coast," in Proc. 7th Eur. Wave and Tidal Energy Conf. (EWTEC), Porto, Portugal, 2007.

[16] M. Folley and T. Whittaker, "The effect of sub-optimal control and the spectral wave climate on the performance of wave energy converter arrays," Appl. Ocean Res., vol. 31, no. 4, pp. 260-266, 2009.

[17] J. Cruz, R. Sykes, P. Siddorn, and R. Taylor, "Estimating the loads and energy yield of arrays of wave energy converters under realistic seas," IET Renew. Power Generat., vol. 4, pp. 488-497, Nov. 2010.

[18] G. P. Thomas and D. V. Evans, "Arrays of three-dimensional waveenergy absorbers," J. Fluid Mechanics, vol. 108, pp. 67-88, Jun. 1981.

[19] J. Westphalen, G. Bacelli, P. Balitsky, and J. V. Ringwood, "Control strategies for arrays of wave energy devices," in Proc. 9th Eur. Wave and Tidal Energy Conf. (EWTEC), Southampton, U.K., 2011.

[20] W. Cummins, "The impulse response function and ship motions," Schiffstechnik, vol. 9, pp. 101-109, 1962.

[21] T. Ogilvie, "Recent progress toward the understanding and prediction of ship motions," in Proc. 5th Symp. Naval Hydrodynamics, Bergen, Norway, 1964.

[22] T. Perez and T. I. Fossen, "Time- vs. frequency-domain identification of parametric radiation force models for marine structures at zero speed," Modeling, Identification and Control, vol. 29, no. 1, pp. 1-19, 2008.

[23] WAMIT Inc [Online]. Available: www.wamit.com

[24] J. F. Douglas, J. Gasiorek, and J. Swaffield, Fluid Mechanics, 5th ed. Englewood Cliffs, NJ, USA: Pearson/Prentice-Hall, 2005.

[25] S. Bellew and T. Stallard, "Linear modelling of wave device arrays and comparison to experimental and second order models," in Proc. Int. Workshop for Water Waves and Floating Bodies, Harbin, China, 2010, pp. 1-4.

[26] M. A. Bhinder, A. Babarit, L. Gentaz, and P. Ferrant, "Assessment of viscous damping via 3D-cfd modelling of a floating wave energy device," in Proc. 9th Eur. Wave and Tidal Energy Conf. (EWTEC), Southampton, U.K., 2011.

[27] G. M. Terra, W. J. van de Berg, and L. R. M. Maas, "Experimental verification of Lorentz' linearization procedure for quadratic friction," Fluid Dynamics Res., vol. 36, pp. 175-188, 1995.

[28] M. Folley, T. Whittaker, and J. van't Hoff, "The design of small seabedmounted bottom-hinged wave energy converters," in Proc. 7th Eur. Wave and Tidal Energy Conf. (EWTEC), Porto, Portugal, 2007.

[29] F. Fusco and J. Ringwood, "A study of the prediction requirements in real-time control of wave energy converters," IEEE Trans. Sustain. Energy, vol. 3, no. 1, pp. 176-184, Jan. 2012.

[30] G. Bacelli, J. V. Ringwood, and J.-C. Gilloteaux, "A control system for a self-reacting point absorber wave energy converter subject to constraints," in IFAC World Congress, Milan, Italy, 2011, vol. 18, pp. $11387-11392$.

[31] H. Kwakernaak and R. Sivan, Linear Optimal Control Systems. Hoboken, NJ, USA: Wiley-Interscience, 1972, vol. 172.
[32] M. Folley and T. Whittaker, "The adequacy of phase-averaged models for modelling wave farms," in Proc. 30th Int. Conf. Ocean, Offshore and Arctic Engineering (OMAE), Rotterdam, The Netherlands, 2011, vol. 5, pp. 663-671, ASME.

[33] A. Babarit, "Impact of long separating distances on the energy production of two interacting wave energy converters," Ocean Eng., vol. 37, no. 8-9, pp. 718-729, 2010.

[34] A. Babarit, B. Borgarino, P. Ferrant, and A. Clément, "Assessment of the influence of the distance between two wave energy converters on energy production," IET Renew. Power Generat., vol. 4, pp. 592-601, Nov. 2010.

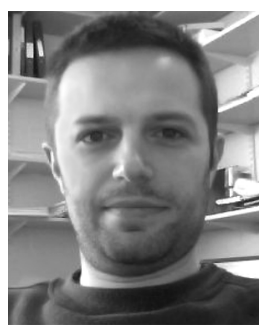

Giorgio Bacelli received the Laurea Magistrale in electronic engineering from Università Politecnica delle Marche, in 2006. He worked on his thesis as a Socrates student at the Electronic Engineering Department in National University of Ireland (NUI), Maynooth, Ireland, on the design of an impedance matching controller for an inductively coupled plasma chamber. In December 2006, he joined the Dynamics and Control Research Group at NUI Maynooth as a Ph.D. student. From December 2006 until June 2007, he worked on the control of semiconductor etching processes. He is completing his $\mathrm{Ph} . \mathrm{D}$. on the modeling and control of wave energy converters at the Centre for Ocean Energy Research at NUI Maynooth.

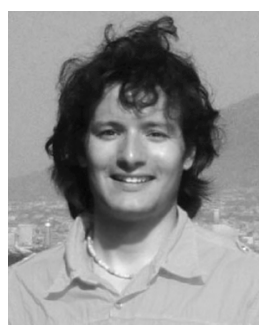

Philip Balitsky received the B.Sc. degree in physics from the University of Virginia, Charlottesville, VA, USA, in 2006, and the M.Sc. degree in physical oceanography from Old Dominion University, Norfolk, VA, USA, in 2008. He recently completed the M.Eng.Sc. degree on control and modeling of arrays of wave energy converters.

While completing his M.Sc. degree, he worked at the USACE Field Research Facility, Duck, NC, USA, on nearshore wave modeling and observation. Following his graduation, he worked at Virginia Polytechnic Institute, Arlington, VA, USA, on a wave energy resource mapping project run by the Electrical Power Research Institute for the U.S. Department of Energy. He joined the Centre for Ocean Research at National University of Ireland (NUI), Maynooth, Ireland, in October 2010.

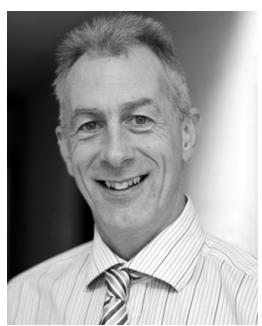

John V. Ringwood (M'87-SM'97) received the Diploma in electrical engineering from Dublin Institute of Technology, in 1981, and the Ph.D. degree in control systems from Strathclyde University, Scotland, in 1985

$\mathrm{He}$ is currently Professor of Electronic Engineering with the National University of Ireland (NUI), Maynooth, Ireland, and Associate Dean for Engineering in the Faculty of Science and Engineering. He was Head of the Electronic Engineering Department at NUI Maynooth from 2000 to 2006, developing the Department from a greenfield site. His research interests cover time series modeling, wave energy, control of plasma processes, and biomedical engineering.

Dr. Ringwood is a Chartered Engineer and a Fellow of the Institution of Engineers of Ireland. 